\title{
New studies on dynamic analysis of asymptotically almost periodic recurrent neural networks involving mixed delays
}

\section{Yuehua Yu' ${ }^{1}$ Shuhua Gong ${ }^{2 *}$ (1) and Zijun Ning ${ }^{2}$}

\section{"Correspondence:}

shuhuagong@aliyun.com ${ }^{2}$ College of Mathematics, Physics and Information Engineering, Jiaxing University, Jiaxing, P.R. China Full list of author information is available at the end of the article

\section{Springer}

\begin{abstract}
This paper studies a class of asymptotically almost periodic recurrent neural networks involving mixed delays. By utilizing differential inequality analysis, some novel assertions are gained to validate the asymptotically almost periodicity of the addressed model, which generalizes and refines some recent literature works. In the end, an example with its numerical simulations is carried out to validate the analytical results.
\end{abstract}

MSC: $34 \mathrm{C} 25 ; 34 \mathrm{~K} 13 ; 34 \mathrm{~K} 25$

Keywords: Recurrent neural network; Asymptotically almost periodicity; Convergence; Mixed delay

\section{Introduction}

In the past forty years, there have been plenty of papers written about the neural network dynamics in various application areas [1-12]. Particularly, (asymptotically, pseudo) almost neural networks have received great deal of attention in the past decade due to their potential applications in classification, associative memory parallel computation, and other fields. So there have been many research results about the almost periodicity [1319], pseudo almost periodicity [20-28], and weighted pseudo almost periodicity [29-32] on neural networks. From the viewpoint of mathematics, let $\left(x_{1}(t), x_{2}(t), \ldots, x_{n}(t)\right)$ represent the state vector, recurrent neural networks (RNNs) involving mixed delays can be described as the following nonlinear dynamic system:

$$
\begin{aligned}
x_{i}^{\prime}(t)= & -a_{i}(t) b_{i}\left(x_{i}(t)\right)+\sum_{j=1}^{n} \alpha_{i j}(t) f_{j}\left(x_{j}(t)\right)+\sum_{j=1}^{n} \beta_{i j}(t) h_{j}\left(x_{j}\left(t-\sigma_{i j}(t)\right)\right) \\
& +\sum_{j=1}^{n} \gamma_{i j}(t) \int_{0}^{+\infty} K_{i j}(\mu) g_{j}\left(x_{j}(t-\mu)\right) d \mu+I_{i}(t), \quad i \in S=\{1,2, \ldots, n\},
\end{aligned}
$$

which includes many kinds of neural networks such as BAM neural networks, Hopfield neural networks, and cellular neural networks. Here the decay function $b_{i}$ and activation functions $f_{j}, g_{j}, h_{j}$ are continuous, $a_{i}(t)$ represents the rate of decay, $I_{i}(t)$ denotes the exter-

(c) The Author(s) 2018. This article is distributed under the terms of the Creative Commons Attribution 4.0 International License (http://creativecommons.org/licenses/by/4.0/), which permits unrestricted use, distribution, and reproduction in any medium, provided you give appropriate credit to the original author(s) and the source, provide a link to the Creative Commons license, and indicate if changes were made. 
nal input. Further information on the mixed delays and coefficient parameters is available from $[1,13,14]$.

Recently, for $b_{i}(u)=u(i \in S)$, by using the exponential dichotomy theorem in semilinear differential systems, the almost periodicity and pseudo almost periodicity have been fully investigated in [15-19] and [20-32], respectively. Nevertheless, as a nonlinear differential equation, RNNs (1.1) involving that $b_{i}(u) \neq u$ for some $i \in S$ has no exponential dichotomy, and there are a few research works on the asymptotically almost periodicity analysis for this case. It is worth pointing out that all results in [13-32] are established under

Assumption (E): $\quad a_{i}(t)$ is almost periodic on $\mathbb{R}$ for all $i \in S$.

Now, a question naturally arises: how about the asymptotically almost periodicity of RNNs (1.1) without assuming $(\mathrm{E})$ and $b_{i}(u)=u(i \in S)$. Inspired by the preceding discussions, in this paper, avoiding $(\mathrm{E})$ and $b_{i}(u)=u(i \in S)$, we derive some novel criteria to validate the existence and convergence of the asymptotically almost periodic solutions of (1.1). Main contributions and innovation points of this paper are threefold. First, a class of asymptotically almost periodic recurrent neural networks involving mixed delays is established. Second, a novel approach to the problem of the existence on asymptotically almost periodic solutions of RNNs (1.1) is presented. Third, improved results on the global exponential attractivity of all solutions of RNNs (1.1) are obtained. Furthermore, our results not only generalize the results in [20-32], but also improve them. In truth, one can view the following Remark 3.1 and Remark 4.1 for extensive information.

The rest of this paper is arranged as follows. Some preliminaries and lemmas are supplied in Sect. 2. In Sect. 3, some novel sufficient conditions are gained to evidence the asymptotically almost periodicity of system (1.1). In Sect. 4, an illustrative example is presented to validate the correctness of the proposed theory. In the end, a brief conclusion is presented to summarize and evaluate our work.

\section{Preliminary results}

Notations For $\mathbb{J} \subseteq \mathbb{R}, C_{0}\left(\mathbb{R}^{+}, \mathbb{J}\right)=\left\{v: v \in C\left(\mathbb{R}^{+}, \mathbb{J}\right), \lim _{t \rightarrow+\infty} v(t)=0\right\}$. We designate the collections of the almost periodic functions and the asymptotically almost periodic functions from $\mathbb{R}$ to $\mathbb{J}$ by $\mathrm{AP}(\mathbb{R}, \mathbb{J})$ and $\mathrm{AAP}\left(\mathbb{R}, \mathbb{J}^{n}\right)$, respectively. For the definitions of $\mathrm{AP}$ and $\mathrm{APP}$, we refer the reader to $[33,34]$. For $i, j \in S$, we suppose that $a_{i}, \sigma_{i j} \in \mathrm{AAP}\left(\mathbb{R}, \mathbb{R}^{+}\right)$, $I_{i}, \alpha_{i j}, \beta_{i j}, \gamma_{i j} \in \operatorname{AAP}(\mathbb{R}, \mathbb{R})$, and

$$
\begin{aligned}
& a_{i}=a_{i}^{0}+a_{i}^{1}, \quad I_{i}=I_{i}^{0}+I_{i}^{1}, \quad \alpha_{i j}=\alpha_{i j}^{0}+\alpha_{i j}^{1}, \\
& \beta_{i j}=\beta_{i j}^{0}+\beta_{i j}^{1}, \quad \gamma_{i j}=\gamma_{i j}^{0}+\gamma_{i j}^{1}, \quad \sigma_{i j}=\sigma_{i j}^{0}+\sigma_{i j}^{1},
\end{aligned}
$$

where $a_{i}^{0}, \sigma_{i j}^{0} \in \mathrm{AP}\left(\mathbb{R}, \mathbb{R}^{+}\right), I_{i}^{0}, \alpha_{i j}^{0}, \beta_{i j}^{0}, \gamma_{i j}^{0} \in \mathrm{AP}(\mathbb{R}, \mathbb{R}), a_{i}^{1}, \sigma_{i j}^{1} \in C_{0}\left(\mathbb{R}^{+}, \mathbb{R}^{+}\right), I_{i}^{1}, \alpha_{i j}^{1}, \beta_{i j}^{1}, \gamma_{i j}^{1} \in$ $C_{0}\left(\mathbb{R}^{+}, \mathbb{R}\right)$.

Assumptions For $i, j \in S$ and $u, v \in \mathbb{R}$, there are constants $\underline{b}_{i}>0, \bar{b}_{i}>0, L_{j}^{f}, L_{j}^{h}, L_{j}^{g}$, $\eta_{1}, \eta_{2}, \ldots, \eta_{n}, \xi$, and $\lambda$ such that

$\left(U_{0}\right) b_{i}(0)=0, \underline{b}_{i}|u-v| \leq \operatorname{sign}(u-v)\left(b_{i}(u)-b_{i}(v)\right) \leq \bar{b}_{i}|u-v|$.

$\left(U_{1}\right)\left|f_{j}(u)-f_{j}(v)\right| \leq L_{j}^{f}|u-v|,\left|h_{j}(u)-h_{j}(v)\right| \leq L_{j}^{h}|u-v|,\left|g_{j}(u)-g_{j}(v)\right| \leq L_{j}^{g}|u-v|$.

$\left(U_{2}\right) K_{i j}: \mathbb{R}^{+} \rightarrow \mathbb{R}$ is continuous and absolutely integrable. 
$\left(U_{3}\right)$

$$
\begin{aligned}
& -\left[a_{i}^{0}(t) \underline{b}_{i}-\lambda\right] \eta_{i}+\sum_{j=1}^{n}\left(\left|\alpha_{i j}^{0}(t)\right|+\left|\alpha_{i j}^{1}(t)\right|\right) L_{j}^{f} \eta_{j}+\sum_{j=1}^{n}\left(\left|\beta_{i j}^{0}(t)\right|+\left|\beta_{i j}^{1}(t)\right|\right) e^{\lambda \sigma} L_{j}^{h} \eta_{j} \\
& \quad+\sum_{j=1}^{n}\left(\left|\gamma_{i j}^{0}(t)\right|+\left|\gamma_{i j}^{1}(t)\right|\right) \int_{0}^{+\infty}\left|K_{i j}(s)\right| e^{\lambda s} d s L_{j}^{g} \eta_{j}<-\xi, \\
& t \in \mathbb{R}^{+}, \sigma=\max _{i, j \in S} \sup _{t \in \mathbb{R}} \sigma_{i j}^{0}(t) .
\end{aligned}
$$

For further analysis, we set up the following nonlinear auxiliary system:

$$
\begin{aligned}
x_{i}^{\prime}(t)= & -a_{i}^{0}(t) b_{i}\left(x_{i}(t)\right)+\sum_{j=1}^{n} \alpha_{i j}^{0}(t) f_{j}\left(x_{j}(t)\right)+\sum_{j=1}^{n} \beta_{i j}^{0}(t) h_{j}\left(x_{j}\left(t-\sigma_{i j}^{0}(t)\right)\right) \\
& +\sum_{j=1}^{n} \gamma_{i j}^{0}(t) \int_{0}^{+\infty} K_{i j}(u) g_{j}\left(x_{j}(t-u)\right) d u+I_{i}^{0}(t), \quad i \in S .
\end{aligned}
$$

The initial condition involved in systems $(1.1)$ and $(1.1)^{0}$ can be described as follows:

$$
x_{i}(s)=\varphi_{i}(s), \quad s \in(-\infty, 0], i \in S, \varphi \text { is bounded and continuous on }(-\infty, 0] .
$$

Denote $\|x\|=\max _{i \in S}\left|x_{i}\right|,\|x(t)\|_{\eta}=\max _{i \in S}\left|\eta_{i}^{-1} x_{i}(t)\right|$, and let $i_{t}$ be such a designation that

$$
\eta_{i_{t}}^{-1}\left|x_{i_{t}}(t)\right|=\|x(t)\|_{\eta}
$$

Lemma 2.1 Designate $x(t)$ to be a solution of the initial value problem $(1.1)^{0}$ and (2.1). If $\left(U_{0}\right),\left(U_{1}\right),\left(U_{2}\right)$, and $\left(U_{3}\right)$ hold, then $x(t)$ is bounded and exists on $[0,+\infty)$.

Proof Denote $\left[0, \eta^{*}(\varphi)\right)$ to be the maximal right existence interval of $x(t)$. Apparently, we can take $N_{\varphi}>0$ such that

$$
\begin{aligned}
N_{\varphi}>1 & +\sup _{t \in(-\infty, 0]}\|\varphi(t)\| \\
& +\max _{i \in S} \sup _{t \in \mathbb{R}}\left[\sum_{j=1}^{n}\left|\alpha_{i j}^{0}(t)\right|\left|f_{j}(0)\right|+\sum_{j=1}^{n}\left|\beta_{i j}^{0}(t)\right|\left|h_{j}(0)\right|\right. \\
& \left.+\sum_{j=1}^{n}\left|\gamma_{i j}^{0}(t)\right| \int_{0}^{+\infty}\left|K_{i j}(\mu)\right| d \mu\left|g_{j}(0)\right|\right]
\end{aligned}
$$

and

$$
\left|x_{i}(t)\right|<\eta_{i} \frac{\left\|I^{0}\right\|_{\infty}+N_{\varphi}}{\xi} \text { for all } t \in(-\infty, 0], i \in S
$$

We claim that

$$
\left|x_{i}(t)\right|<\eta_{i} \frac{\left\|I^{0}\right\|_{\infty}+N_{\varphi}}{\xi} \quad \text { for all } t \in\left[0, \eta^{*}(\varphi)\right), i \in S .
$$


Suppose the contrary and choose $i \in S$ and $t^{*} \in\left(0, \eta^{*}(\varphi)\right)$ such that

$$
\begin{aligned}
& \left|x_{i}\left(t^{*}\right)\right|=\eta_{i} \frac{\left\|I^{0}\right\|_{\infty}+N_{\varphi}}{\xi}, \quad \text { and } \\
& \left|x_{j}(t)\right|<\eta_{j} \frac{\left\|I^{0}\right\|_{\infty}+N_{\varphi}}{\xi} \text { for all } t \in\left(-\infty, t^{*}\right), j \in S .
\end{aligned}
$$

It follows from $\left(U_{0}\right),\left(U_{1}\right),\left(U_{2}\right),\left(U_{3}\right)$, and (2.4) that

$$
\begin{aligned}
& 0 \leq D^{-}\left(\left|x_{i}\left(t^{*}\right)\right|\right) \\
& \leq-a_{i}^{0}\left(t^{*}\right)\left|b_{i}\left(x_{i}\left(t^{*}\right)\right)\right|+\mid \sum_{j=1}^{n} \alpha_{i j}^{0}\left(t^{*}\right) f_{j}\left(x_{j}\left(t^{*}\right)\right)+\sum_{j=1}^{n} \beta_{i j}^{0}\left(t^{*}\right) h_{j}\left(x_{j}\left(t^{*}-\sigma_{i j}^{0}\left(t^{*}\right)\right)\right) \\
& +\sum_{j=1}^{n} \gamma_{i j}^{0}\left(t^{*}\right) \int_{0}^{+\infty} K_{i j}(\mu) g_{j}\left(x_{j}\left(t^{*}-\mu\right)\right) d \mu+I_{i}^{0}\left(t^{*}\right) \\
& \leq-a_{i}^{0}\left(t^{*}\right) \underline{b}_{i} \eta_{i} \frac{\left\|I^{0}\right\|_{\infty}+N_{\varphi}}{\xi}+\sum_{j=1}^{n}\left|\alpha_{i j}^{0}\left(t^{*}\right)\right|\left(\left|f_{j}\left(x_{j}\left(t^{*}\right)\right)-f_{j}(0)\right|+\left|f_{j}(0)\right|\right) \\
& +\sum_{j=1}^{n}\left|\beta_{i j}^{0}\left(t^{*}\right)\right|\left(\left|h_{j}\left(x_{j}\left(t^{*}-\sigma_{i j}^{0}\left(t^{*}\right)\right)\right)-h_{j}(0)\right|+\left|h_{j}(0)\right|\right) \\
& +\sum_{j=1}^{n}\left|\gamma_{i j}^{0}\left(t^{*}\right)\right| \int_{0}^{+\infty}\left|K_{i j}(\mu)\right|\left(\left|g_{j}\left(x_{j}\left(t^{*}-\mu\right)\right)-g_{j}(0)\right|+\left|g_{j}(0)\right|\right) d \mu+\left|I_{i}^{0}\left(t^{*}\right)\right| \\
& \leq-a_{i}^{0}\left(t^{*}\right) \underline{b}_{i} \eta_{i} \frac{\left\|I^{0}\right\|_{\infty}+N_{\varphi}}{\xi}+\sum_{j=1}^{n}\left|\alpha_{i j}^{0}\left(t^{*}\right)\right| L_{j}^{f} \eta_{j} \frac{\left\|I^{0}\right\|_{\infty}+N_{\varphi}}{\xi} \\
& +\sum_{j=1}^{n}\left|\beta_{i j}^{0}\left(t^{*}\right)\right| L_{j}^{h} \eta_{j} \frac{\left\|I^{0}\right\|_{\infty}+N_{\varphi}}{\xi} \\
& +\sum_{j=1}^{n}\left|\gamma_{i j}^{0}\left(t^{*}\right)\right| \int_{0}^{+\infty}\left|K_{i j}(\mu)\right| d \mu L_{j}^{g} \eta_{j} \frac{\left\|I^{0}\right\|_{\infty}+N_{\varphi}}{\xi} \\
& +\sum_{j=1}^{n}\left|\alpha_{i j}^{0}\left(t^{*}\right)\right|\left|f_{j}(0)\right|+\sum_{j=1}^{n}\left|\beta_{i j}^{0}\left(t^{*}\right)\right|\left|h_{j}(0)\right| \\
& +\sum_{j=1}^{n}\left|\gamma_{i j}^{0}\left(t^{*}\right)\right| \int_{0}^{+\infty}\left|K_{i j}(\mu)\right| d \mu\left|g_{j}(0)\right|+\left|I_{i}^{0}\left(t^{*}\right)\right| \\
& <\left[-a_{i}^{0}\left(t^{*}\right) \underline{b}_{i} \eta_{i}+\sum_{j=1}^{n}\left|\alpha_{i j}^{0}\left(t^{*}\right)\right| L_{j}^{f} \eta_{j}+\sum_{j=1}^{n}\left|\beta_{i j}^{0}\left(t^{*}\right)\right| L_{j}^{h} \eta_{j}\right. \\
& \left.+\sum_{j=1}^{n}\left|\gamma_{i j}^{0}\left(t^{*}\right)\right| \int_{0}^{+\infty}\left|K_{i j}(\mu)\right| d \mu L_{j}^{g} \eta_{j}\right] \frac{\left\|I^{0}\right\|_{\infty}+N_{\varphi}}{\xi}+\left\|I^{0}\right\|_{\infty}+N_{\varphi} \\
& <0 \text {, }
\end{aligned}
$$

which derives a contradiction and proves the above claim. Thus, the boundedness and the extension theorem of solution in [35] entail that $\eta^{*}(\varphi)=+\infty$, which finishes the proof of Lemma 2.1 . 
Remark 2.1 Under the assumptions in Lemma 2.1, an argument similar to that applied in Lemma 2.1 shows that each solution of initial value problem (1.1) and (2.1) is bounded on $[0,+\infty)$.

Lemma 2.2 Let $\left(U_{0}\right),\left(U_{1}\right),\left(U_{2}\right)$, and $\left(U_{3}\right)$ hold. Suppose that $x(t)$ is a solution of system $(1.1)^{0}$ with the initial function $\varphi$ satisfying (2.1), and $\varphi^{\prime}$ is bounded and continuous on $(-\infty, 0]$. Then, for any $\epsilon>0$, one can pick a relatively dense subset $M_{\epsilon}$ in $\mathbb{R}$ to satisfy that, for any $\tau \in M_{\epsilon}$, there is $N=N(\tau)>0$ obeying

$$
\|x(t+\tau)-x(t)\|_{\eta}<\frac{\epsilon}{2 \max _{i \in S} \eta_{i}} \text { for all } t>N
$$

Proof Denote

$$
\begin{aligned}
P_{i}(\tau, t) & \\
= & -\left[a_{i}^{0}(t+\tau)-a_{i}^{0}(t)\right] b_{i}\left(x_{i}(t+\tau)\right)+\sum_{j=1}^{n}\left[\alpha_{i j}^{0}(t+\tau)-\alpha_{i j}^{0}(t)\right] f_{j}\left(x_{j}(t+\tau)\right) \\
& +\sum_{j=1}^{n}\left[\beta_{i j}^{0}(t+\tau)-\beta_{i j}^{0}(t)\right] h_{j}\left(x_{j}\left(t-\sigma_{i j}^{0}(t+\tau)+\tau\right)\right) \\
& +\sum_{j=1}^{n} \beta_{i j}^{0}(t)\left[h_{j}\left(x_{j}\left(t-\sigma_{i j}^{0}(t+\tau)+\tau\right)\right)-h_{j}\left(x_{j}\left(t-\sigma_{i j}^{0}(t)+\tau\right)\right)\right] \\
& +\sum_{j=1}^{n}\left[\gamma_{i j}^{0}(t+\tau)-\gamma_{i j}^{0}(t)\right] \int_{0}^{+\infty} K_{i j}(\mu) g_{j}\left(x_{j}(t+\tau-\mu)\right) d \mu+\left[I_{i}^{0}(t+\tau)-I_{i}^{0}(t)\right] .
\end{aligned}
$$

According to Lemma 2.1 and the boundedness of $x(t)$, one finds that $x(t)$ is uniformly continuous on $\mathbb{R}$. Thus, for any $\epsilon>0$, one can take $0<\epsilon^{*}<\epsilon$ to obey that

$$
\left.\begin{array}{llr}
\left|a_{i}^{0}(t)-a_{i}^{0}(t+\tau)\right|<\epsilon^{*}, & \left|I_{i}^{0}(t)-I_{i}^{0}(t+\tau)\right|<\epsilon^{*}, \quad\left|\sigma_{i j}^{0}(t)-\sigma_{i j}^{0}(t+\tau)\right|<\epsilon^{*}, \\
\left|\alpha_{i j}^{0}(t)-\alpha_{i j}^{0}(t+\tau)\right|<\epsilon^{*}, \quad & \left|\beta_{i j}^{0}(t)-\beta_{i j}^{0}(t+\tau)\right|<\epsilon^{*}, \quad\left|\gamma_{i j}^{0}(t)-\gamma_{i j}^{0}(t+\tau)\right|<\epsilon^{*},
\end{array}\right\}
$$

suggests that

$$
\left|P_{i}(\tau, t)\right|<\frac{1}{2 \max _{i \in S} \eta_{i}} \xi \epsilon,
$$

where $t \in \mathbb{R}, i, j \in S$.

Note that $\left\{a_{i}^{0}, I_{i}^{0}, \alpha_{i j}^{0}, \beta_{i j}^{0}, \gamma_{i j}^{0}, \sigma_{i j}^{0} \in \mathrm{AP}(\mathbb{R}, \mathbb{R})(i, j \in S)\right\}$ is a uniformly almost periodic family. From Corollary 2.3 in [33, p. 19], one can pick a relatively dense subset $M_{\epsilon^{*}}$ in $\mathbb{R}$ to satisfy that

$$
\begin{aligned}
& \left|a_{i}^{0}(t)-a_{i}^{0}(t+\tau)\right|<\epsilon^{*}, \quad\left|I_{i}^{0}(t)-I_{i}^{0}(t+\tau)\right|<\epsilon^{*}, \\
& \left.\left|\alpha_{i j}^{0}(t)-\alpha_{i j}^{0}(t+\tau)\right|<\epsilon^{*}, \quad\left|\sigma_{i j}^{0}(t)-\sigma_{i j}^{0}(t+\tau)\right|<\epsilon^{*},\right\} \quad \tau \in M_{\epsilon^{*}}, t \in \mathbb{R} . \\
& \left|\beta_{i j}^{0}(t)-\beta_{i j}^{0}(t+\tau)\right|<\epsilon^{*}, \quad\left|\gamma_{i j}^{0}(t)-\gamma_{i j}^{0}(t+\tau)\right|<\epsilon^{*},
\end{aligned}
$$

Denote $M_{\epsilon}=M_{\epsilon^{*}}$, for each $\tau \in M_{\epsilon}$, (2.6) and (2.7) give us

$$
\left|P_{i}(\tau, t)\right|<\frac{1}{2 \max _{i \in S} \eta_{i}} \xi \epsilon \quad \text { for all } t \in \mathbb{R}, i \in S .
$$


Designate $t>T_{0}=1+\max \{0,-\tau\}$ and $z_{i}(t)=x_{i}(t+\tau)-x_{i}(t)$, one can obtain

$$
\begin{aligned}
\frac{d z_{i}(t)}{d t}= & -a_{i}^{0}(t)\left[b_{i}\left(x_{i}(t+\tau)\right)-b_{i}\left(x_{i}(t)\right)\right]+\sum_{j=1}^{n} \alpha_{i j}^{0}(t)\left[f_{j}\left(x_{j}(t+\tau)\right)-f_{j}\left(x_{j}(t)\right)\right] \\
& +\sum_{j=1}^{n} \beta_{i j}^{0}(t)\left[h_{j}\left(x_{j}\left(t-\sigma_{i j}^{0}(t)+\tau\right)\right)-h_{j}\left(x_{j}\left(t-\sigma_{i j}^{0}(t)\right)\right)\right] \\
& +\sum_{j=1}^{n} \gamma_{i j}^{0}(t) \int_{0}^{+\infty} K_{i j}(\mu)\left[g_{j}\left(x_{j}(t+\tau-\mu)\right)-g_{j}\left(x_{j}(t-\mu)\right)\right] d \mu+P_{i}(\tau, t),
\end{aligned}
$$

and

$$
\begin{aligned}
\left.D^{-}\left(e^{\lambda s}\left|z_{i_{s}}(s)\right|\right)\right|_{s=t} & \\
\leq & \lambda e^{\lambda t}\left|z_{i_{t}}(t)\right|+e^{\lambda t}\left\{-a_{i_{t}}^{0}(t)\left|b_{i_{t}}\left(x_{i_{t}}(t+\tau)\right)-b_{i_{t}}\left(x_{i_{t}}(t)\right)\right|\right. \\
& +\mid \sum_{j=1}^{n} \alpha_{i_{t} j}^{0}(t)\left[f_{j}\left(x_{j}(t+\tau)\right)-f_{j}\left(x_{j}(t)\right)\right] \\
& +\sum_{j=1}^{n} \beta_{i_{t} j}^{0}(t)\left[h_{j}\left(x_{j}\left(t-\sigma_{i_{t} j}^{0}(t)+\tau\right)\right)-h_{j}\left(x_{j}\left(t-\sigma_{i_{t} j}^{0}(t)\right)\right)\right] \\
& \left.+\sum_{j=1}^{n} \gamma_{i_{t} j}^{0}(t) \int_{0}^{\infty} K_{i_{t} j}(\mu)\left[g_{j}\left(x_{j}(t+\tau-\mu)\right)-g_{j}\left(x_{j}(t-\mu)\right)\right] d \mu+P_{i_{t}}(\tau, t) \mid\right\} \\
\leq & e^{\lambda t}\left\{-\left[a_{i_{t}}^{0}(t) \underline{b}_{i_{t}}-\lambda\right]\left|z_{i_{t}}(t)\right| \eta_{i_{t}}^{-1} \eta_{i_{t}}+\sum_{j=1}^{n} \alpha_{i_{t} j}^{0}(t) L_{j}^{f}\left|z_{j}(t)\right| \eta_{j}^{-1} \eta_{j}\right. \\
& +\sum_{j=1}^{n} \beta_{i_{t} j}^{0}(t) L_{j}^{h}\left|z_{j}\left(t-\sigma_{i_{t} j}^{0}(t)\right)\right| \eta_{j}^{-1} \eta_{j} \\
& \left.+\sum_{j=1}^{n} \gamma_{i_{t j}}^{0}(t) \int_{0}^{+\infty}\left|K_{i_{t} j}(\mu)\right| L_{j}^{g}\left|z_{j}(t-\mu)\right| \eta_{j}^{-1} \eta_{j} d \mu\right\}+e^{\lambda t}\left|P_{i_{t}}(\tau, t)\right| .
\end{aligned}
$$

Denote

$$
Q(t)=\sup _{s \leq t}\left\{e^{\lambda s}\|z(s)\|_{\eta}\right\} \quad \text { for all } t \geq T_{0} .
$$

Obviously, $Q(t)$ is nondecreasing.

If $Q(t)-e^{\lambda t}|z(t)|$ is eventually positive, then one can pick $T_{1}>T_{0}$ satisfying

$$
Q(t)>e^{\lambda t}\|z(t)\|_{\eta} \quad \text { for all } t \geq T_{1} .
$$

Then, for each $t \geq T_{1}$, there exists $\varepsilon_{t}>0$ such that

$$
Q(t)>e^{\lambda s}\|z(s)\|_{\eta} \quad \text { for all } s \in\left(t-\varepsilon_{t}, t+\varepsilon_{t}\right)
$$


and

$$
Q(t) \equiv Q(s) \quad \text { for all } s \in\left(t-\varepsilon_{t}, t+\varepsilon_{t}\right) \text {. }
$$

Therefore,

$$
Q(t) \equiv Q\left(T_{1}\right) \quad \text { is a constant for all } t \geq T_{1},
$$

and there is $T_{2}>T_{1}$ satisfying

$$
\|z(t)\|_{\eta} \leq e^{-\lambda t} Q(t)=e^{-\lambda t} Q\left(T_{1}\right)<\frac{\epsilon}{2 \max _{i \in S} \eta_{i}} \quad \text { for all } t \geq T_{2}
$$

If $Q(t)-e^{\lambda t}|z(t)|$ is not eventually positive, then $A=\left\{t \geq T_{0}: Q(t)=e^{\lambda t}\|z(t)\|_{\eta}\right\} \cap$ $[s,+\infty) \neq \varnothing$ for all $s \geq T_{0}$. Take $T^{t} \geq T_{0}$ satisfying $Q\left(T^{t}\right)=e^{\lambda T^{t}}\left\|z\left(T^{t}\right)\right\|_{\eta},\left(U_{3}\right)$ and (2.9) yield

$$
\begin{aligned}
0 \leq & \left.D^{+}\left(e^{\lambda s}\left|z_{i_{s}}(s)\right|\right)\right|_{s=T^{t}} \\
\leq & e^{\lambda T^{t}}\left\{-\left[a_{i_{T^{t}}}^{0}\left(T^{t}\right) \underline{b}_{T_{T^{t}}}-\lambda\right]\left|z_{i_{T^{t}}}\left(T^{t}\right)\right| \eta_{i_{T^{t}}}^{-1} \eta_{i_{T^{t}}}+\sum_{j=1}^{n} \alpha_{i_{T^{t}}}^{0}\left(T^{t}\right) L_{j}^{f}\left|z_{j}\left(T^{t}\right)\right| \eta_{j}^{-1} \eta_{j}\right. \\
& +\sum_{j=1}^{n} \beta_{i_{T^{t}}}^{0}\left(T^{t}\right) L_{j}^{h}\left|z_{j}\left(T^{t}-\sigma_{i_{T^{t}}}^{0}\left(T^{t}\right)\right)\right| \eta_{j}^{-1} \eta_{j} \\
& \left.+\sum_{j=1}^{n} \gamma_{i_{T^{t}} j}^{0}\left(T^{t}\right) \int_{0}^{+\infty}\left|K_{i_{T^{t}} j}(\mu)\right| L_{j}^{g}\left|z_{j}\left(T^{t}-\mu\right)\right| \eta_{j}^{-1} \eta_{j} d \mu\right\}+e^{\lambda T^{t}}\left|P_{i_{T^{t}}}\left(\tau, T^{t}\right)\right| \\
\leq & \left\{-\left[a_{i_{T^{t}}}^{0}\left(T^{t}\right) \underline{b}_{i_{T^{t}}}-\lambda\right] \eta_{i_{T^{t}}}+\sum_{j=1}^{n} \alpha_{i_{T^{t j}}}^{0}\left(T^{t}\right) L_{j}^{f} \eta_{j}\right. \\
& +\sum_{j=1}^{n} \beta_{i_{T^{t}}^{0}}\left(T^{t}\right) L_{j}^{h} e^{\lambda \sigma} \eta_{j} \\
& \left.+\sum_{j=1}^{n} \gamma_{i_{T^{t}} j}\left(T^{t}\right) \int_{0}^{+\infty}\left|K_{i_{T^{t}}}(\mu)\right| e^{\lambda \mu} d \mu L_{j}^{g} \eta_{j}\right\} Q\left(T^{t}\right)+e^{\lambda T^{t}}\left|P_{i_{T^{t}}}\left(\tau, T^{t}\right)\right| \\
\leq & -\xi Q\left(T^{t}\right)+e^{\lambda T^{t}}\left|P_{i_{T^{t}}}\left(\tau, T^{t}\right)\right| .
\end{aligned}
$$

Hence, (2.8) and (2.10) lead to

$$
\left\|z\left(T^{t}\right)\right\|_{\eta} \leq e^{-\lambda T^{t}} Q\left(T^{t}\right) \leq e^{-\lambda T^{t}} e^{\lambda T^{t}} \frac{1}{\xi}\left|P_{i_{T}}\left(\tau, T^{t}\right)\right|<\frac{\epsilon}{2 \max _{i \in S} \eta_{i}} .
$$

Similarly, one can derive that $\|z(\chi)\|_{\eta}<\frac{\epsilon}{2 \max _{i \in S} \eta_{i}}$ provided that $\chi>T^{t}$ with $Q(\chi)=$ $e^{\lambda \chi}\|z(\chi)\|_{\eta}$. Therefore, assuming that $t>T^{t}$ and $Q(t)>e^{\lambda t}|z(t)|$, one can take $T_{*}^{t} \in\left[T^{t}, t\right)$ satisfying

$$
Q\left(T_{*}^{t}\right)=e^{\lambda T_{*}^{t}}\left\|z\left(T_{*}^{t}\right)\right\|_{\eta}, \quad \text { and } \quad Q(s)>e^{\lambda s}\|z(s)\|_{\eta} \quad \text { for all } s \in\left(T_{*}^{t}, t\right] \text {. }
$$


From the fact that $\left\|z\left(T_{*}^{t}\right)\right\|_{\eta}<\frac{\epsilon}{2 \max _{i \in S} \eta_{i}}$, we get

$$
Q(s) \equiv Q\left(T_{*}^{t}\right) \quad \text { for all } s \in\left(T_{*}^{t}, t\right], \quad \text { and } \quad\|z(t)\|_{\eta} \leq e^{-\lambda t} e^{\lambda T_{*}^{t}}\left\|z\left(T_{*}^{t}\right)\right\|_{\eta}<\frac{\epsilon}{2 \max _{i \in S} \eta_{i}}
$$

Finally, there is $N=N(\tau)>0$ satisfying that

$$
\|z(t)\|_{\eta}<\frac{\epsilon}{2 \max _{i \in S} \eta_{i}} \quad \text { for all } t>N
$$

This finishes the proof of Lemma 2.2.

\section{Asymptotically almost periodicity}

Theorem 3.1 If $\left(U_{0}\right),\left(U_{1}\right),\left(U_{2}\right)$, and $\left(U_{3}\right)$ hold, then every solution of $(1.1)$ with initial condition (2.1) is asymptotically almost periodic on $\mathbb{R}^{+}$and converges to an almost periodic function $x^{*}(t)$ as $t \rightarrow+\infty$, which is a unique almost periodic solution of system $(1.1)^{0}$.

Proof Denote $u(t)=\left(u_{1}(t), u_{2}(t), \ldots, u_{n}(t)\right)$ to be a solution of system $(1.1)^{0}$ in Lemma 2.2, and

$$
\begin{aligned}
P_{i, q}( & \\
= & -\left[a_{i}^{0}\left(t+t_{q}\right)-a_{i}^{0}(t)\right] b_{i}\left(u_{i}\left(t+t_{q}\right)\right)+\sum_{j=1}^{n}\left[\alpha_{i j}^{0}\left(t+t_{q}\right)-\alpha_{i j}^{0}(t)\right] f_{j}\left(u_{j}\left(t+t_{q}\right)\right) \\
& +\sum_{j=1}^{n}\left[\beta_{i j}^{0}\left(t+t_{q}\right)-\beta_{i j}^{0}(t)\right] h_{j}\left(u_{j}\left(t-\sigma_{i j}^{0}\left(t+t_{q}\right)+t_{q}\right)\right) \\
& +\sum_{j=1}^{n} \beta_{i j}^{0}(t)\left[h_{j}\left(u_{j}\left(t-\sigma_{i j}^{0}\left(t+t_{q}\right)+t_{q}\right)\right)-h_{j}\left(u_{j}\left(t-\sigma_{i j}^{0}(t)+t_{q}\right)\right)\right] \\
& +\sum_{j=1}^{n}\left[\gamma_{i j}^{0}\left(t+t_{q}\right)-\gamma_{i j}^{0}(t)\right] \int_{0}^{+\infty} K_{i j}(\mu) g_{j}\left(u_{j}\left(t+t_{q}-\mu\right)\right) d \mu \\
& +\left[I_{i}^{0}\left(t+t_{q}\right)-I_{i}^{0}(t)\right], \quad i \in S,
\end{aligned}
$$

where $\left\{t_{q}\right\}_{q \geq 1} \subseteq \mathbb{R}$ is a sequence. Then

$$
\begin{aligned}
u_{i}^{\prime}(t+ & \left.t_{q}\right) \\
= & -a_{i}^{0}(t) b_{i}\left(u_{i}\left(t+t_{q}\right)\right)+\sum_{j=1}^{n} \alpha_{i j}^{0}(t) f_{j}\left(u_{j}\left(t+t_{q}\right)\right)+\sum_{j=1}^{n} \beta_{i j}^{0}(t) h_{j}\left(u_{j}\left(t-\sigma_{i j}^{0}(t)+t_{q}\right)\right) \\
& +\sum_{j=1}^{n} \gamma_{i j}^{0}(t) \int_{0}^{+\infty} K_{i j}(\mu) g_{j}\left(u_{j}\left(t+t_{q}-\mu\right)\right) d \mu \\
& +I_{i}^{0}(t)+P_{i, q}(t), \quad i \in S, t+t_{q} \geq 0 .
\end{aligned}
$$

In a similar way to the proof of (2.8), we can take $\left\{t_{q}\right\}_{q \geq 1}$ satisfying

$$
\left|P_{i, q}(t)\right|<\frac{1}{q} \quad \text { for all } i, q, t \text {. }
$$


Note that $\left\{u\left(t+t_{q}\right)\right\}_{q \geq 1}$ is uniformly bounded and equiuniformly continuous, from the Arzela-Ascoli lemma, one can select a subsequence $\left\{t_{q_{j}}\right\}_{j \geq 1}$ of $\left\{t_{q}\right\}_{q \geq 1}$ to satisfy that $\left\{u\left(t+t_{q_{j}}\right)\right\}_{j \geq 1}$ (we also designate it by $\left\{u\left(t+t_{q}\right)\right\}_{q \geq 1}$ ) is convergent uniformly to a bounded and continuous function $x^{*}(t)=\left(x_{1}^{*}(t), x_{2}^{*}(t), \ldots, x_{n}^{*}(t)\right)$ in any compact set of $\mathbb{R}$. Consequently,

$$
\begin{aligned}
& a_{i}^{0}(t) h_{i}\left(u_{i}\left(t+t_{q}\right)\right) \quad \Rightarrow \quad a_{i}^{0}(t) h_{i}\left(x_{i}^{*}(t)\right), \\
& \sum_{j=1}^{n} \alpha_{i j}^{0}(t) f_{j}\left(u_{j}\left(t+t_{q}\right)\right) \quad \Rightarrow \quad \sum_{j=1}^{n} \alpha_{i j}^{0}(t) f_{j}\left(x_{j}^{*}(t)\right) \text {, } \\
& \left.\sum_{j=1}^{n} \beta_{i j}^{0}(t) h_{j}\left(u_{j}\left(t-\sigma_{i j}^{0}(t)+t_{q}\right)\right) \quad \Rightarrow \quad \sum_{j=1}^{n} \beta_{i j}^{0}(t) h_{j}\left(x_{j}^{*}\left(t-\sigma_{i j}^{0}(t)\right)\right),\right\}
\end{aligned}
$$$$
\text { as } q \rightarrow+\infty
$$

in every compact set of $\mathbb{R}$. Here, the symbol " $\Rightarrow$ " represents " is convergent uniformly to". Now, we show that

$$
\begin{gathered}
\sum_{j=1}^{n} \gamma_{i j}(t) \int_{0}^{+\infty} K_{i j}(\mu) g_{j}\left(u_{j}\left(t+t_{q}-\mu\right)\right) d \mu \\
\Rightarrow \sum_{j=1}^{n} \gamma_{i j}(t) \int_{0}^{+\infty} K_{i j}(\mu) g_{j}\left(x_{j}^{*}(t-\mu)\right) d \mu \\
(q \rightarrow+\infty) \text { on any compact set of } \mathbb{R} .
\end{gathered}
$$

For any $\varepsilon>0$ and $[a, b] \subseteq \mathbb{R},\left(U_{2}\right)$ and the boundedness of $u$ and $x^{*}$ entail that one can pick $A^{*}>0$ to satisfy that

$$
\begin{aligned}
& \mid \sum_{j=1}^{n} \gamma_{i j}(t) \int_{A^{*}}^{+\infty} K_{i j}(\mu) g_{j}\left(u_{j}\left(t+t_{q}-\mu\right)\right) d \mu \\
& \quad-\sum_{j=1}^{n} \gamma_{i j}(t) \int_{A^{*}}^{+\infty} K_{i j}(\mu) g_{j}\left(x_{j}^{*}(t-\mu)\right) d \mu \mid<\frac{\varepsilon}{2}
\end{aligned}
$$

for all $i, t, q$. Note that $\left\{u\left(t+t_{q}\right)\right\}$ is convergent uniformly to $x^{*}(t)$ on $\left[a-A^{*}, b\right]$, one can take a positive integer $q^{*}$ to satisfy that, for $q>q^{*}$ and $t \in[a, b]$,

$$
\left|\sum_{j=1}^{n} \gamma_{i j}(t) \int_{0}^{A^{*}} K_{i j}(\mu) g_{j}\left(u_{j}\left(t+t_{q}-\mu\right)\right) d u-\sum_{j=1}^{n} \gamma_{i j}(t) \int_{0}^{A^{*}} K_{i j}(\mu) g_{j}\left(x_{j}^{*}(t-\mu)\right) d \mu\right|<\frac{\varepsilon}{2} .
$$

This and (3.5) produce that

$$
\begin{aligned}
& \left|\sum_{j=1}^{n} \gamma_{i j}(t) \int_{0}^{+\infty} K_{i j}(\mu) g_{j}\left(u_{j}\left(t+t_{q}-\mu\right)\right) d \mu-\sum_{j=1}^{n} \gamma_{i j}(t) \int_{0}^{+\infty} K_{i j}(\mu) g_{j}\left(x_{j}^{*}(t-\mu)\right) d \mu\right| \\
& \quad<\varepsilon, \quad \text { for all } q>q^{*}, t \in[a, b],
\end{aligned}
$$


which leads to (3.4). Hence, (3.1), (3.2), (3.3), and (3.4) suggest that $\left\{u_{i}^{\prime}\left(t+t_{q}\right)\right\}_{q \geq 1}$ is convergent uniformly to

$$
\begin{aligned}
& -a_{i}^{0}(t) h_{i}\left(x_{i}^{*}(t)\right)+\sum_{j=1}^{n} \alpha_{i j}^{0}(t) f_{j}\left(x_{j}^{*}(t)\right) \\
& +\sum_{j=1}^{n} \beta_{i j}^{0}(t) h_{j}\left(x_{j}^{*}\left(t-\sigma_{i j}^{0}(t)\right)\right)+\sum_{j=1}^{n} \gamma_{i j}(t) \int_{0}^{+\infty} K_{i j}(\mu) g_{j}\left(x_{j}^{*}(t-\mu)\right) d \mu+I_{i}^{0}(t)
\end{aligned}
$$

on every compact set in $\mathbb{R}$. Furthermore, one can derive that $x^{*}(t)$ is a solution of $(1.1)^{0}$ and

$$
\begin{aligned}
\left(x_{i}^{*}(t)\right)^{\prime}= & -a_{i}^{0}(t) h_{i}\left(x_{i}^{*}(t)\right)+\sum_{j=1}^{n} \alpha_{i j}^{0}(t) f_{j}\left(x_{j}^{*}(t)\right)+\sum_{j=1}^{n} \beta_{i j}^{0}(t) h_{j}\left(x_{j}^{*}\left(t-\sigma_{i j}^{0}(t)\right)\right) \\
& +\sum_{j=1}^{n} \gamma_{i j}(t) \int_{0}^{+\infty} K_{i j}(\mu) g_{j}\left(x_{j}^{*}(t-\mu)\right) d \mu+I_{i}^{0}(t) \quad \text { for all } t \in \mathbb{R}, i \in S .
\end{aligned}
$$

Hereafter, for any $\epsilon>0$, according to Lemma 2.2, one can pick a relatively dense subset $M_{\epsilon}$ in $\mathbb{R}$ such that, for any $\tau \in M_{\epsilon}$, there is $N=N(\tau)>0$ obeying

$$
\left|u_{i}\left(s+t_{q}+\tau\right)-u_{i}\left(s+t_{q}\right)\right| \leq \eta_{i}\left\|u\left(s+t_{q}+\tau\right)-u\left(s+t_{q}\right)\right\|_{\eta}<\frac{\epsilon}{2} \quad \text { for all } s+t_{q}>N,
$$

and

$$
\lim _{q \rightarrow+\infty}\left|u_{i}\left(s+t_{q}+\tau\right)-u_{i}\left(s+t_{q}\right)\right|=\left|x_{i}^{*}(s+\tau)-x_{i}^{*}(s)\right| \leq \frac{\epsilon}{2}<\epsilon \quad \text { for all } s \in \mathbb{R}, i \in S,
$$

which, together with definitions of AP in $[33,34]$, proves that $x^{*}(t)$ is an almost periodic solution of $(1.1)^{0}$.

Now, let $x(t)$ be an arbitrary solution of the initial value problem (1.1) and (2.1), we turn to demonstrate that $\lim _{t \rightarrow+\infty} x(t)=x^{*}(t)$. Set $y(t)=\left\{y_{j}(t)\right\}=\left\{x_{j}(t)-x_{j}^{*}(t)\right\}=x(t)-x^{*}(t)$ and

$$
\begin{aligned}
B_{i}(t)= & -a_{i}^{1}(t) b_{i}\left(x_{i}(t)\right)+\sum_{j=1}^{n} \alpha_{i j}^{1}(t) f_{j}\left(x_{j}(t)\right)+\sum_{j=1}^{n} \beta_{i j}^{1}(t) h_{j}\left(x_{j}\left(t-\sigma_{i j}(t)\right)\right) \\
& +\sum_{j=1}^{n} \beta_{i j}^{0}(t)\left[h_{j}\left(x_{j}\left(t-\sigma_{i j}(t)\right)\right)-h_{j}\left(x_{j}\left(t-\sigma_{i j}^{0}(t)\right)\right)\right] \\
& +\sum_{j=1}^{n} \gamma_{i j}^{1}(t) \int_{0}^{+\infty} K_{i j}(\mu) g_{j}\left(x_{j}(t-\mu)\right) d \mu+I_{i}^{1}(t), \quad i \in S .
\end{aligned}
$$

Thus

$$
\begin{aligned}
y_{i}^{\prime}(t)= & -a_{i}^{0}(t)\left[b_{i}\left(x_{i}(t)\right)-b_{i}\left(x_{i}^{*}(t)\right)\right]+\sum_{j=1}^{n} \alpha_{i j}^{0}(t)\left(f_{j}\left(y_{j}(t)+x_{j}^{*}(t)\right)-f_{j}\left(x_{j}^{*}(t)\right)\right) \\
& +\sum_{j=1}^{n} \beta_{i j}^{0}(t)\left(h_{j}\left(y_{j}\left(t-\sigma_{i j}^{0}(t)\right)+x_{j}^{*}\left(t-\sigma_{i j}^{0}(t)\right)\right)-h_{j}\left(x_{j}^{*}\left(t-\sigma_{i j}^{0}(t)\right)\right)\right)
\end{aligned}
$$




$$
\begin{aligned}
& +\sum_{j=1}^{n} \gamma_{i j}^{0}(t) \int_{0}^{+\infty} K_{i j}(\mu)\left(g_{j}\left(y_{j}(t-\mu)+x_{j}^{*}(t-\mu)\right)-g_{j}\left(x_{j}^{*}(t-\mu)\right)\right) d \mu \\
& +B_{i}(t), \quad i \in S .
\end{aligned}
$$

Since $a_{i}^{1}, \sigma_{i j}^{1} \in C_{0}\left(\mathbb{R}^{+}, \mathbb{R}^{+}\right), I_{i}^{1}, \alpha_{i j}^{1}, \beta_{i j}^{1}, \gamma_{i j}^{1} \in C_{0}\left(\mathbb{R}^{+}, \mathbb{R}\right)$ and $x$ is uniformly continuous on $\mathbb{R}$, one can take a constant $T_{0}^{\varphi}>0$ to satisfy that, for every $\epsilon>0$,

$$
\left|B_{i}(t)\right|<\xi \frac{\epsilon}{2 \max _{i \in S} \eta_{i}} \quad \text { for all } t>T_{0}^{\varphi}, i \in S,
$$

and

$$
\begin{aligned}
\left.D^{-}\left(e^{\lambda s}\left|y_{i_{s}}(s)\right|\right)\right|_{s=t} & \\
\leq & e^{\lambda t}\left\{-\left[a_{i_{t}}^{0}(t) \underline{b}_{i_{t}}-\lambda\right]\left|y_{i_{t}}(t)\right| \eta_{i_{t}}^{-1} \eta_{i_{t}}+\sum_{j=1}^{n} \alpha_{i_{t} j}^{0}(t) L_{j}^{f}\left|y_{j}(t)\right| \eta_{j}^{-1} \eta_{j}\right. \\
& +\sum_{j=1}^{n} \beta_{i_{i j}}^{0}(t) L_{j}^{h}\left|y_{j}\left(t-\sigma_{i_{t} j}^{0}(t)\right)\right| \eta_{j}^{-1} \eta_{j} \\
& \left.+\sum_{j=1}^{n} \gamma_{i_{t j}}^{0}(t) \int_{0}^{+\infty}\left|K_{i_{t} j}(\mu)\right| L_{j}^{g}\left|y_{j}(t-\mu)\right| \eta_{j}^{-1} \eta_{j} d \mu\right\}+e^{\lambda t}\left|B_{i_{t}}(t)\right| \text { for all } t>T_{0}^{\varphi} .
\end{aligned}
$$

Define

$$
\Gamma(t)=\sup _{s \leq t}\left\{e^{\lambda s}\|y(s)\|_{\eta}\right\} \quad \text { for all } t \in \mathbb{R}
$$

Then, an argument similar to that used in Lemma 2.2 shows that there exists $T^{\varphi} \geq T_{0}^{\varphi}$ satisfying

$$
\|y(t)\|_{\eta}<\frac{\epsilon}{2 \max _{i \in S} \eta_{i}} \quad \text { for all } t \geq T^{\varphi},
$$

which implies

$$
\lim _{t \rightarrow+\infty} x(t)=x^{*}(t), \quad \text { and } \quad x(t) \in \operatorname{AAP}\left(\mathbb{R}, \mathbb{R}^{n}\right) .
$$

Therefore, $(1.1)^{0}$ has a unique almost periodic solution $N^{*}(t)$. The proof is finished.

Remark 3.1 Under the conditions in Lemma 2.2, from Lemma 2.1 and Lemma 2.2, by applying a similar way as that in Theorem 3.1 of [13], one can show that every solution $x(t)$ of $(1.1)^{0}$ converges exponentially to $x^{*}(t)$ as $t \rightarrow+\infty$. Since $\mathrm{AP}(\mathbb{R}, \mathbb{R})$ is a proper subspace of $\operatorname{AAP}(\mathbb{R}, \mathbb{R})$, one can easily see that all the results on $(1.1)^{0}$ in [13] are special ones of Theorem 3.1 in this paper. Most recently, the authors in [36] established asymptotically almost periodicity on shunting inhibitory cellular neural networks with time-varying delays and continuously distributed delays. However, the asymptotically almost periodicity on recurrent neural networks without the assumption $E$ and the condition $b_{i}(u)=u$ has not been explored in [36]. This implies that Theorem 3.1 generalizes and complements the main results of $[13,36]$. 


\section{A numerical example}

Example 4.1 Regard the following asymptotically almost periodic recurrent neural networks:

$$
\left\{\begin{aligned}
x_{1}^{\prime}(t)= & -\left(10+\cos \sqrt{2} t+\frac{1}{1+|t|}\right)\left(20 x_{1}(t)+\arctan x_{1}(t)\right) \\
& +\left(\cos \sqrt{3} t+\frac{1}{|t|+3}\right) f_{1}\left(x_{1}(t)\right)+\left(\cos \sqrt{3} t+\frac{1}{|t|+3}\right) f_{2}\left(x_{2}(t)\right) \\
& +\left(\cos \sqrt{5} t+\frac{1}{|t|+2}\right) h_{1}\left(x_{1}(t-2)\right)+\left(\cos \sqrt{5} t+\frac{1}{|t|+2}\right) h_{2}\left(x_{2}(t-2)\right) \\
& +\left(\cos \sqrt{7} t+\frac{1}{|t|+2}\right) \int_{0}^{+\infty} e^{-2 \mu} g_{1}\left(x_{1}(t-\mu)\right) d \mu+(\cos \sqrt{7} t \\
& \left.+\frac{1}{|t|+2}\right) \int_{0}^{+\infty} e^{-2 \mu} g_{2}\left(x_{2}(t-\mu)\right) d \mu+100 \sin t+\frac{1}{5|t|+1} \\
x_{2}^{\prime}(t)= & -\left(10+\sin \sqrt{2} t+\frac{1}{1+|t|}\right)\left(30 x_{2}(t)+\arctan x_{2}(t)\right) \\
& +\left(\cos \sqrt{11} t+\frac{1}{|t|+3}\right) f_{1}\left(x_{1}(t)\right)+\left(\cos \sqrt{11} t+\frac{1}{|t|+3}\right) f_{2}\left(x_{2}(t)\right) \\
& +\left(\cos \sqrt{15} t+\frac{1}{|t|+2}\right) h_{1}\left(x_{1}(t-2)\right)+\left(\cos \sqrt{15} t+\frac{1}{|t|+2}\right) h_{2}\left(x_{2}(t-2)\right) \\
& +\left(\cos \sqrt{17} t+\frac{1}{|t|+2}\right) \int_{0}^{+\infty} e^{-2 \mu} g_{1}\left(x_{1}(t-\mu)\right) d \mu+(\cos \sqrt{17} t \\
& \left.+\frac{1}{|t|+2}\right) \int_{0}^{+\infty} e^{-2 \mu} g_{2}\left(x_{2}(t-\mu)\right) d \mu+100 \cos t+\frac{1}{25|t|+1}
\end{aligned}\right.
$$

Here $h_{1}(x)=h_{2}(x)=\frac{1}{20} \arctan x, f_{1}(x)=f_{2}(x)=g_{1}(x)=g_{2}(x)=\frac{1}{20} x$,

$$
\begin{aligned}
& a_{1}(t)=(10+\cos \sqrt{2} t)+\frac{1}{1+|t|}, \quad a_{2}(t)=(10+\sin \sqrt{2} t)+\frac{1}{1+|t|}, \\
& b_{1}(u)=(20 u+\arctan u), \quad b_{2}(u)=(30 u+\arctan u)
\end{aligned}
$$

and

$$
\begin{aligned}
\left(\begin{array}{ll}
\alpha_{11}(t) & \alpha_{12}(t) \\
\alpha_{21}(t) & \alpha_{22}(t)
\end{array}\right) & =\left(\begin{array}{ll}
\left(\cos \sqrt{3} t+\frac{1}{|t|+3}\right) & \left(\cos \sqrt{3} t+\frac{1}{|t|+3}\right) \\
\left(\cos \sqrt{11} t+\frac{1}{|t|+3}\right) & \left(\cos \sqrt{11} t+\frac{1}{|t|+3}\right)
\end{array}\right), \\
\left(\begin{array}{ll}
\beta_{11}(t) & \beta_{12}(t) \\
\beta_{21}(t) & \beta_{22}(t)
\end{array}\right) & =\left(\begin{array}{ll}
\left(\cos \sqrt{5} t+\frac{1}{|t|+2}\right) & \left(\cos \sqrt{5} t+\frac{1}{|t|+2}\right) \\
\left(\cos \sqrt{15} t+\frac{1}{|t|+2}\right) & \left(\cos \sqrt{15} t+\frac{1}{|t|+2}\right)
\end{array}\right), \\
\left(\begin{array}{ll}
\gamma_{11}(t) & \gamma_{12}(t) \\
\gamma_{21}(t) & \gamma_{22}(t)
\end{array}\right) & =\left(\begin{array}{ll}
\left(\cos \sqrt{7} t+\frac{1}{|t|+2}\right) & \left(\cos \sqrt{7} t+\frac{1}{|t|+2}\right) \\
\left(\cos \sqrt{17} t+\frac{1}{|t|+2}\right) & \left(\cos \sqrt{17} t+\frac{1}{|t|+2}\right)
\end{array}\right) .
\end{aligned}
$$

Let $\eta_{i}=1, L_{j}^{f}=L_{j}^{h}=L_{j}^{g}=\frac{1}{20}, \underline{b}_{1}=20, \underline{b}_{2}=30, \xi=5, i, j=1,2$, we can see that system (4.1) obeys all the conditions in Theorem 3.1. Therefore, each solution of (4.1) is convergent to the same almost periodic function as $t \rightarrow+\infty$, which is also an asymptotically almost periodic function on $\mathbb{R}^{+}$. This fact can be revealed in Fig. 1: Numerical solutions of system (4.1) with initial values $(10,-30),(-30,40),(30,-60)$, respectively.

Remark 4.1 Clearly,

$$
a_{1}(t)=(10+\cos \sqrt{2} t)+\frac{1}{1+|t|} \quad \text { and } \quad a_{2}(t)=(10+\sin \sqrt{2} t)+\frac{1}{1+|t|}
$$

are not almost periodic functions, and $b_{1}(u)=(20 u+\arctan u)$ and $b_{2}(u)=(30 u+\arctan u)$ do not satisfy that $b_{i}(u)=u(i \in S)$. Thus, all the results established in [13-32, 36] cannot be 


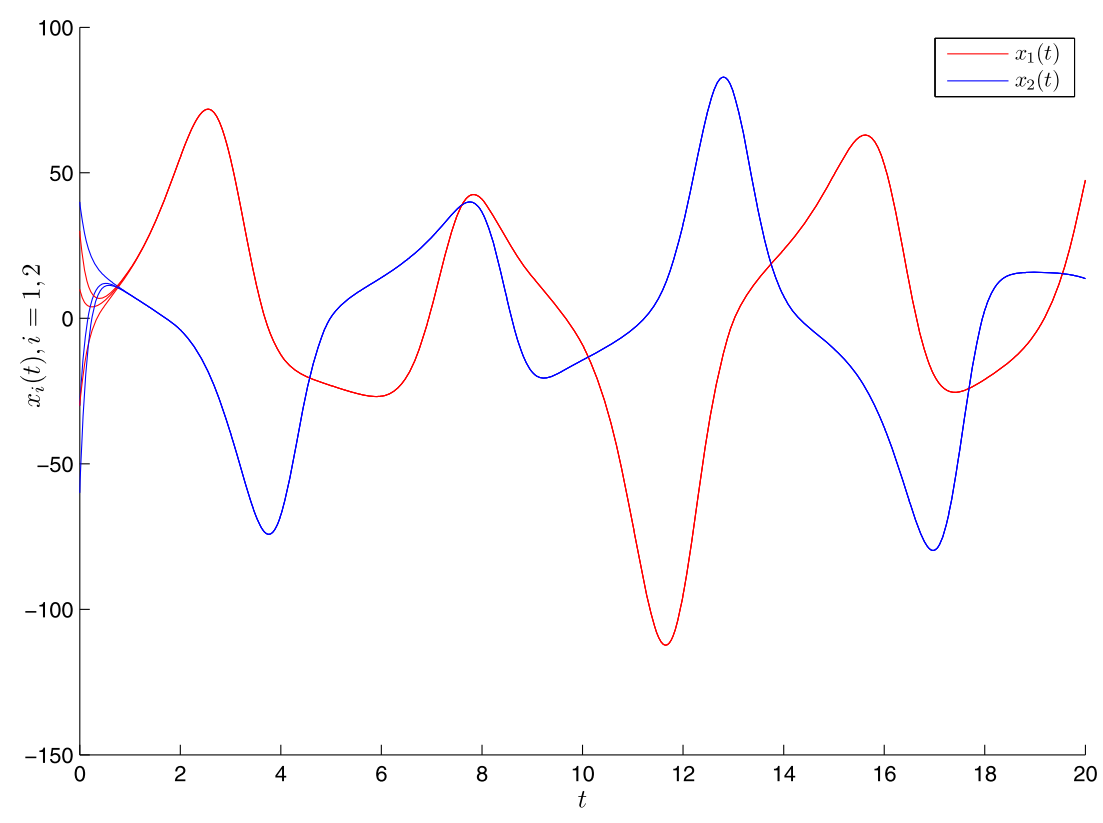

Figure 1 Numerical solutions of system (4.1) with different initial values

applied to imply that all the solutions of (4.1) converge globally to the almost periodic solution. On the other hand, to the best of the authors' knowledge, there is no research work concerning the asymptotically almost periodicity on recurrent neural networks without the assumption $E$ and the condition $b_{i}(u)=u$. Therefore, the results established in this paper are essentially new.

\section{Conclusions}

In this paper, avoiding the exponential dichotomy theory, the asymptotically almost periodicity on recurrent neural networks involving mixed delays has been explored. By combining the Lyapunov function method with differential inequality approach, some sufficient assertions have been gained to validate the global convergence of the addressed model. Particularly, our conditions are easily checked in practice by simple inequality methods, and the approach adopted in this paper provides a possible way to research the topic on asymptotically almost periodic dynamics of other nonlinear neural network models. In future research, we will research the dynamics for asymptotically almost periodic Cohen-Grossberg neural network models.

\section{Acknowledgements}

The authors would like to express their sincere appreciation to the editors and anonymous reviewers for their constructive comments and suggestions which helped them to improve the present paper. This work was supported by Zhejiang Provincial Natural Science Foundation of China (Grant Nos. LY16A010018, LY18A010019), Zhejiang Provincial Education Department Natural Science Foundation of China (Y201533862), and Natural Scientific Research Fund of Hunan Provincial Education Department of China (Grant No. 17C1076).

\section{Funding}

This work was supported by Zhejiang Provincial Natural Science Foundation of China (Grant Nos. LY16A010018, LY18A010019), Zhejiang Provincial Education Department Natural Science Foundation of China (Y201533862), and Natural Scientific Research Fund of Hunan Provincial Education Department of China (Grant No. 17C1076). 
Authors' contributions

YHY, SHG, and ZJN worked together in the derivation of the mathematical results. All authors read and approved the final manuscript.

\section{Author details}

${ }^{1}$ College of Mathematics and Computer Science, Hunan University of Arts and Science, Changde, P.R. China. ${ }^{2}$ College of Mathematics, Physics and Information Engineering, Jiaxing University, Jiaxing, P.R. China.

\section{Publisher's Note}

Springer Nature remains neutral with regard to jurisdictional claims in published maps and institutional affiliations.

\section{Received: 25 June 2018 Accepted: 5 November 2018 Published online: 14 November 2018}

\section{References}

1. Wu, J: Introduction to Neural Dynamics and Signal Trasmission Delay. de Gruyter, Belin (2001)

2. Huang, C., Yang, Z., Yi, T., Zou, X.: On the basins of attraction for a class of delay differential equations with non-monotone bistable nonlinearities. J. Differ. Equ. 256(7), 2101-2114 (2014)

3. Huang, C., Cao, J., Cao, J. D.: Stability analysis of switched cellular neural networks: A mode-dependent average dwell time approach. Neural Netw. 82, 84-99 (2016)

4. Arik, S., Orman, Z.: Global stability analysis of Cohen-Grossberg neural networks with time-varying delays. Phys. Lett. A 341, 410-421 (2005)

5. Huang, C., Liu, B.: New studies on dynamic analysis of inertial neural networks involving non-reduced order method. Neurocomputing 325, 283-287 (2019)

6. Chen, T., Lu, W., Chen, G.: Dynamical behaviors of a large class of general delayed neural networks. Neural Comput. 17, 949-968 (2005)

7. Chen, Z.: Global exponential stability of anti-periodic solutions for neutral type CNNs with D operator. Int. J. Mach. Learn. Cybern. 9(7), 1109-1115 (2018). https://doi.org/10.1007/s13042-016-0633-9

8. Jia, R.: Finite-time stability of a class of fuzzy cellular neural networks with multi-proportional delays. Fuzzy Sets Syst. 319(15), 70-80 (2017)

9. Yang, G.: New results on convergence of fuzzy cellular neural networks with multi-proportional delays. Int. J. Mach. Learn. Cybern. 9(10), 1675-1682 (2018). https://doi.org/10.1007/s13042-017-0672-x

10. Yao, L.: Dynamics of Nicholson's blowflies models with a nonlinear density-dependent mortality. Appl. Math. Model. 64, 185-195 (2018)

11. Jiang, A.: Exponential convergence for HCNNs with oscillating coefficients in leakage terms. Neural Process. Lett. 43 , 285-294 (2016)

12. Long, Z:: New results on anti-periodic solutions for SICNNs with oscillating coefficients in leakage terms. Neurocomputing 171(1), 503-509 (2016)

13. Liu, B., Huang, L.: Positive almost periodic solutions for recurrent neural networks. Nonlinear Anal., Real World Appl. 9 , 830-841 (2008)

14. Lu, W., Chen, T.: Global exponential stability of almost periodic solutions for a large class of delayed dynamical systems. Sci. China Ser. A 8(48), 1015-1026 (2005)

15. $\mathrm{Xu}, \mathrm{Y}$ :: New results on almost periodic solutions for CNNs with time-varying leakage delays. Neural Comput. Appl. 25, 1293-1302 (2014)

16. Zhang, H., Shao, J.: Existence and exponential stability of almost periodic solutions for CNNs with time-varying leakage delays. Neurocomputing 121(9), 226-233 (2013)

17. Zhang, H., Shao, J: Almost periodic solutions for cellular neural networks with time-varying delays in leakage terms. Appl. Math. Comput. 219(24), 11471-11482 (2013)

18. Zhang, H.: Existence and stability of almost periodic solutions for CNNs with continuously distributed leakage delays. Neural Comput. Appl. 2014(24), 1135-1146 (2014)

19. Zhang, A.: Almost periodic solutions for SICNNs with neutral type proportional delays and D operators. Neural Process. Lett. 47(1) 57-70 (2018). https://doi.org/10.1007/s11063-017-9631-5

20. Liu, B., Tunc, C.: Pseudo almost periodic solutions for CNNs with leakage delays and complex deviating arguments. Neural Comput. Appl. 26, 429-435 (2015)

21. Liu, B.: Pseudo almost periodic solutions for neutral type CNNs with continuously distributed leakage delays. Neurocomputing 148, 445-454 (2015)

22. Liang, J., Qian, H., Liu, B.: Pseudo almost periodic solutions for fuzzy cellular neural networks with multi-proportional delays. Neural Process. Lett. 48, 1201-1212 (2018)

23. Zhang, A.: Pseudo almost periodic solutions for SICNNs with oscillating leakage coefficients and complex deviating arguments. Neural Process. Lett. 45, 183-196 (2017)

24. Zhang, A.: Pseudo almost periodic solutions for neutral type SICNNs with D operator. J. Exp. Theor. Artif. Intell. 29(4), 795-807 (2017)

25. Zhang, A.: Pseudo almost periodic solutions for CNNs with oscillating leakage coefficients and complex deviating arguments. J. Exp. Theor. Artif. Intell. (2017). https://doi.org/10.1080/0952813X.2017.1354084

26. Zhang, A.: Pseudo almost periodic high-order cellular neural networks with complex deviating arguments. Int. J. Mach. Learn. Cybern. 30(1), 89-100 (2018). https://doi.org/10.1007/s13042-017-0715-3

27. Tang, Y.: Pseudo almost periodic shunting inhibitory cellular neural networks with multi-proportional delays. Neura Process. Lett. 48(1), 167-177 (2018). https://doi.org/10.1007/s11063-017-9708-1

28. Xu, Y.: Exponential stability of pseudo almost periodic solutions for neutral type cellular neural networks with $D$ operator. Neural Process. Lett. 46, 329-342 (2017). https://doi.org/10.1007/s11063-017-9584-8

29. Zhou, Q.: Weighted pseudo anti-periodic solutions for cellular neural networks with mixed delays. Asian J. Contro 19(4), 1557-1563 (2017) 
30. Zhou, Q., Shao, J.: Weighted pseudo anti-periodic SICNNs with mixed delays. Neural Comput. Appl. 29(10), 865-872 (2018). https://doi.org/10.1007/s00521-016-2582-3

31. Xu, Y.: Weighted pseudo-almost periodic delayed cellular neural networks. Neural Comput. Appl. 30(8), 2453-2458 (2018). https://doi.org/10.1007/s00521-016-2820-8

32. $\mathrm{Xu}, \mathrm{Y}$.: Exponential stability of weighted pseudo almost periodic solutions for HCNNs with mixed delays. Neural Process. Lett. 46, 507-519 (2017)

33. Zhang, C.: Almost Periodic Type Functions and Ergodicity. Kluwer Academic, Beijing (2003)

34. Fink, A.M.: Almost Periodic Differential Equations. Lecture Notes in Mathematics, vol. 377, pp. 80-112. Springer, Berlin (1974)

35. Hino, Y., Murakami, S., Naito, T.: Functional Differential Equations with Infinite Delay, Lecture Notes in Mathematics, vol. 1473, pp. 338-352. Springer, Berlin (1985)

36. Huang, C., Liu, B., Tian, X., et al.: Global convergence on asymptotically almost periodic SICNNs with nonlinear decay functions. Neural Process. Lett. (2018). https://doi.org/10.1007/s11063-018-9835-3

\section{Submit your manuscript to a SpringerOpen ${ }^{\circ}$} journal and benefit from:

- Convenient online submission

$\checkmark$ Rigorous peer review

- Open access: articles freely available online

- High visibility within the field

- Retaining the copyright to your article

Submit your next manuscript at $\gg$ springeropen.com 